\title{
Lebenserhaltende Intensivmassnahmen bei extrem frühgeborenen Kindern
}

Unter Neonatologen in industrialisierten Ländern bestehen grosse Meinungsunterschiede, ab welchem Gestationsalter bei extrem frühgeborenen Kindern auf lebenserhaltende Intensivmassnahmen verzichtet werden sollte. So zeigte vor kurzem eine Umfrage in Europa, dass bei einem Frühgeborenen von 24 vollendeten Schwangerschaftswochen (SSW) und 560 g Geburtsgewicht in Italien, Deutschland und Grossbritannien über 90\% der befragten Neonatologen eine Reanimation und Intensivbehandlung beginnen würden, in den Niederlanden aber nur 35\% und in der Schweiz 70\% [1]. Wäre das Kind weniger als 24 Schwangerschaftswochen alt, würden in der Schweiz nur noch 11\% der Ärztinnen und Ärzte unmittelbar nach der Geburt Reanimationsmassnahmen einleiten.

Wie weit die Wünsche der Eltern bei solchen Entscheidungen berücksichtigt werden sollen, ist ebenfalls umstritten. In Europa besteht ein Nord-Süd-Gefälle, indem Ärzte in Grossbritannien, Schweden und den Niederlanden eher bereit sind, dem Wunsch der Eltern zu entsprechen, auch wenn dieser Wunsch von der eigenen Überzeugung abweicht [2]. In Süd- und Osteuropa ist dies weniger der Fall.

In der Schweiz war das Meinungsspektrum unter Neonatologen diesbezüglich nicht so breit wie in Europa, aber doch beachtlich. Aus diesem Grunde setzte die Schweizerische Gesellschaft für Neonatologie eine Arbeitsgruppe ein, die Empfehlungen zur Betreuung von Frühgeborenen an der Grenze der Lebensfähigkeit erarbeiten sollte. Diese bestand aus Neonatologen (Pädiatern), Geburtshelfern und Entwicklungsneurologen. Es wurden die verfügbaren Daten über die Lebensqualität nach Gestationsalter analysiert und Handlungsleitlinien formuliert, die einen individuellen Interpretationsspielraum lassen, aber auch eine (Gestationsalters-)Grenze festlegen, unter der Intensivmassnahmen nicht indiziert sind.

Diese Empfehlungen sind in dreifacher Hinsicht bemerkenswert. Erstens ist es gelungen, sich auf nationaler Ebene einen Konsens zu fin- den. Zweitens weichen diese Empfehlungen klar von denen anderer Länder ab. So setzen die deutschen Leitlinien die Interventionsgrenze eine bis zwei Schwangerschaftswochen tiefer an [3]. Drittens sind diese Empfehlungen wegweisend, indem für eine definierte Patientengruppe, nämlich Frühgeborene, die vor 24 SSW auf die Welt kommen, der Palliativmedizin gegenüber der Intensivmedizin der Vorrang gegeben wird. Diese von mehreren medizinischen Fachgesellschaften gebilligten Empfehlungen sollten dazu führen, dass in der Schweiz medizinisch-ethische Entscheidungen bei Frühgeborenen an der Grenze zur Lebensfähigkeit vereinheitlicht und damit das ethische Prinzip der Gerechtigkeit besser erfüllt wird. Die Schweizerische Akademie der Medizinischen Wissenschaften unterstützt diese Empfehlungen und es ist zu hoffen, dass sie auch von einer breiten Öffentlichkeit zur Kenntnis genommen werden.

Prof. Dr. med. H. U. Bucher

Präsident der Schweizerischen Gesellschaft für Neonatologie

Prof. Dr. med. P. Hohlfeld

Präsident der Schweizerischen Gesellschaft

für Gynäkologie und Geburtshilfe

1 Cuttini M, Nadai M, Kaminski M, Hansen G, de Leeuw R, Lenoir S, et al. End-of-life decisions in neonatal intensive care: physicians' self-reported practices in seven European countries. EURONIC Study Group. Lancet 2000;355:2112-8.

2 Cuttini M, Rebagliato M, Bortoli P, Hansen G, de Leeuw R, Lenoir S, et al. Parental visiting, communication, and participation in ethical decisions: a comparison of neonatal unit policies in Europe. Arch Dis Child Fetal Neonatal Ed 1999;81:F84-91.

3 Frühgeburt an der Grenze der Lebensfähigkeit des Kindes. Gemeinsame Empfehlung der Deutschen Gesellschaft für Gynäkologie und Geburtshilfe, der Deutschen Gesellschaft für Kinderheilkunde und Jugendmedizin, der Deutschen Gesellschaft für Perinatale Medizin und Gesellschaft für Neonatologie und Pädiatrische Intensivmedizin. http://www.uni-duesseldorf.de/WWW/AWMF/ll/ pneon-19.htm. 